\title{
Anomalous quartic gauge boson couplings at hadron colliders
}

\author{
O. J. P. Éboli, ${ }^{1}, *$ M. C. Gonzalez-García, ${ }^{2}$ S. M. Lietti, ${ }^{1}$ and S. F. Novaes ${ }^{1}$ \\ ${ }^{1}$ Instituto de Física Teórica, Universidade Estadual Paulista, Rua Pamplona 145, 01405-900, São Paulo, Brazil \\ ${ }^{2}$ Instituto de Física Corpuscular IFIC CSIC, Universidad de Valencia, Edificio Institutos de Paterna, Apartado 2085 , \\ 46071 Valencia, Spain
}

(Received 22 September 2000; published 7 March 2001)

\begin{abstract}
We analyze the potential of the Fermilab Tevatron and CERN Large Hadron Collider (LHC) to study anomalous quartic vector-boson interactions $\gamma \gamma Z Z$ and $\gamma \gamma W^{+} W^{-}$. Working in the framework of $S U(2)_{L}$ $\otimes U(1)_{Y}$ chiral Lagrangians, we study the production of photon pairs accompanied by $l^{+} l^{-}, l^{ \pm} \nu$, and jet pairs to impose bounds on these new couplings, taking into account the unitarity constraints. We compare our findings with the indirect limits coming from precision electroweak measurements as well as with presently available direct searches at CERN LEPII. We show that the Tevatron run II can provide limits on these quartic limits which are of the same order of magnitude as the existing bounds from LEPII searches. LHC will be able to tighten considerably the direct constraints on these possible new interactions, leading to more stringent limits than the presently available indirect ones.
\end{abstract}

DOI: 10.1103/PhysRevD.63.075008

PACS number(s): 12.60.Cn

\section{INTRODUCTION AND FORMALISM}

Within the framework of the standard model (SM), the structure of the trilinear and quartic vector boson couplings is completely determined by the $S U(2)_{L} \times U(1)_{Y}$ gauge symmetry. The study of these interactions can either lead to an additional confirmation of the model or give some indication of the existence of new phenomena at a higher scale [1]. Presently, the triple gauge-boson couplings are being probed at the Fermilab Tevatron [2] and CERN $e^{+} e^{-}$collider LEP [3] through the production of vector boson pairs; however, we have only started to study the quartic gaugeboson couplings [4,5].

It is important to independently measure the trilinear and quartic gauge-boson couplings because there are extensions of the SM [6] that leave the trilinear couplings unchanged but modify the quartic vertices. A simple way to generate, at the tree level, new quartic gauge-boson interactions is, for instance, by the exchange of a heavy boson between vector boson pairs.

The phenomenological studies of the anomalous vertices $\gamma \gamma W^{+} W^{-}$and $\gamma \gamma Z Z$ have already been carried out for $\gamma \gamma$ $[7,8]$, e $\gamma$ [9], and $e^{+} e^{-}[10]$ colliders. Some preliminary estimates of the potential of the Tevatron collider have also been presented in Ref. [11] where only the effect on the total cross section for "neutral" final states $\gamma W^{+} W^{-}$and $\gamma \gamma Z$ were considered while the most promising charged final state $\gamma \gamma W^{ \pm}$was not included. In this paper we analyze the potential of hadron colliders to unravel deviations on the quartic vector boson couplings by examining the most relevant processes which are the production of two photons accompanied by a lepton pair, where the fermions are produced by the decay of either a $W^{ \pm}$or a $Z^{0}$ in the anomalous contribution, i.e.,

*Present address. Instituto de Física da USP, C.P. 66.318, São Paulo, SP 05389-970, Brazil.

$$
\begin{aligned}
& p+p(\bar{p}) \rightarrow \gamma+\gamma+\left(W^{*} \rightarrow\right) l+\nu, \\
& p+p(\bar{p}) \rightarrow \gamma+\gamma+\left(Z^{*} \rightarrow\right) l+l
\end{aligned}
$$

as well as the production of photon pairs accompanied by jets

$$
p+p \rightarrow \gamma+\gamma+j+j
$$

for the CERN Large Hadron Collider (LHC).

We carry out a detailed analysis of these reactions taking into account the full SM background leading to the same final state. We introduce realistic cuts in order to reduce this background and we include the effect of detector efficiencies in the evaluation of the attainable limits. We further consider the energy dependence (form factor) of the anomalous couplings in order to comply with the unitarity bounds. Our results show that although the analysis of Tevatron run I data can only provide limits on these quartic couplings, which are worse than the existing bounds from LEPII searches, the Tevatron run II could yield bounds of the same order of magnitude as the present LEPII limits. Moreover, the LHC will be able to considerably tighten the direct constraints on these possible new interactions, giving rise to limits more stringent than the presently available indirect bounds.

In order to perform a model independent analysis, we use a chiral Lagrangian to parametrize the anomalous $\gamma \gamma W^{+} W^{-}$ and $\gamma \gamma Z Z$ interactions [12]. Assuming that there is no Higgs boson in the low energy spectrum we employ a nonlinear representation of the spontaneously broken $S U(2)_{L}$ $\otimes U(1)_{Y}$ gauge symmetry. To construct such a lagrangian, it is useful to define the matrix-valued scalar field $\xi(x)$ $=\exp \left(2 i X_{a} \varphi^{a}(x) / v\right)$, where $X_{a}$ are the broken generators and $\varphi^{a}$ are the Nambu-Goldstone bosons of the global symmetrybreaking pattern $S U(2)_{L} \otimes U(1)_{Y} \rightarrow U(1)_{\mathrm{em}}$. We denote the unbroken generator by $Q$ and our conventions are such that $\operatorname{Tr}\left(X_{a} X_{b}\right)=\frac{1}{2} \delta_{a b}$ and $\operatorname{Tr}\left(X_{a} Q\right)=0$.

The action of a transformation $G$ of the gauge group $S U(2)_{L} \otimes U(1)_{Y}$ on $\xi$ takes the form 


$$
\xi \rightarrow \xi^{\prime}, \quad \text { where } \quad G \xi=\xi^{\prime} H^{\dagger} .
$$

$H=\exp (i Q u)$ is defined requiring that $\xi^{\prime}$ contains only the broken generators. In order to write the effective Lagrangian for the gauge bosons, it is convenient to introduce the auxiliary quantity

$$
\mathcal{D}_{\mu}(\xi) \equiv \xi^{\dagger} \partial_{\mu} \xi-i \xi^{\dagger}\left(g W_{\mu}^{a} T_{a}+g^{\prime} B_{\mu} Y\right) \xi,
$$

where $T_{a}$ and $Y$ are the generators of $S U(2)_{L}$ and $U(1)_{Y}$, respectively.

Now we can easily construct fields which have a simple transformation law under $S U(2)_{L} \otimes U(1)_{Y}$ :

$$
\begin{gathered}
e \mathcal{A}_{\mu} \equiv \operatorname{Tr}\left[Q \mathcal{D}_{\mu}(\xi)\right] \quad e \mathcal{A}_{\mu} \rightarrow e \mathcal{A}_{\mu}+\partial_{\mu} u, \\
\sqrt{g^{2}+g^{\prime} \mathcal{Z}_{\mu}} \equiv \operatorname{Tr}\left[X_{3} \mathcal{D}_{\mu}(\xi)\right] \quad \mathcal{Z}_{\mu} \rightarrow \mathcal{Z}_{\mu}, \\
g \mathcal{W}_{\mu}^{ \pm} \equiv i \sqrt{2} \operatorname{Tr}\left[T_{\mp} \mathcal{D}_{\mu}(\xi)\right] \quad \mathcal{W}_{\mu}^{ \pm} \rightarrow e^{ \pm i u Q} \mathcal{W}_{\mu}^{ \pm},
\end{gathered}
$$

with the standard definition $T_{+}=T_{1} \pm i T_{2}$. Notice that the fields $\mathcal{A}, \mathcal{Z}$, and $\mathcal{W}^{ \pm}$transform only electromagnetically under $S U(2)_{L} \otimes U(1)_{Y}$. Therefore, effective Lagrangians must be invariant exclusively under the unbroken $U(1)_{\mathrm{em}}$. Moreover, in the unitary gauge $(\xi=1)$ we have that $\mathcal{A}$ $\rightarrow A, \mathcal{Z} \rightarrow Z$, and $\mathcal{W}^{ \pm} \rightarrow W^{ \pm}$.

Requiring $C$ and $P$ invariance, the lowest order effective interactions involving photons is

$$
\begin{aligned}
\mathcal{L}_{\text {eff }}= & -\frac{\pi \alpha \beta_{1}}{2} F^{\mu \nu} F_{\mu \nu} \mathcal{W}^{+\alpha} \mathcal{W}_{\alpha}^{-}-\frac{\pi \alpha \beta_{2}}{4} F^{\mu \nu} F_{\mu \nu} \mathcal{Z}^{\alpha} \mathcal{Z}_{\alpha} \\
& -\frac{\pi \alpha \beta_{3}}{4} F^{\mu \alpha} F_{\mu \beta}\left(\mathcal{W}_{\alpha}^{+} \mathcal{W}^{-\beta}+\mathcal{W}_{\beta}^{+} \mathcal{W}^{-\alpha}\right) \\
& -\frac{\pi \alpha \beta_{4}}{4} F^{\mu \alpha} F_{\mu \beta} \mathcal{Z}_{\alpha} \mathcal{Z}^{\beta} .
\end{aligned}
$$

In order to avoid the strong low energy constraints coming from the $\rho$ parameter we impose the custodial $S U(2)$ symmetry which leads to $\beta_{1}=c_{W}^{2} \beta_{2}=\beta_{0}$ and $\beta_{3}=c_{W}^{2} \beta_{4}=\beta_{c}$. With this choice $\mathcal{L}_{\text {eff }}$ reduces to the parametrization used in Ref. [7]. In the unitary gauge, Eq. (9) gives rise to anomalous $\gamma \gamma Z Z$ and $\gamma \gamma W^{+} W^{-}$vertices which are related by the custodial symmetry.

\section{PRESENT CONSTRAINTS: PRECISION DATA, LEPII, AND UNITARITY BOUNDS}

The couplings defined in the effective Lagrangian Eq. (9) contribute at the one-loop level to the $Z$ physics [9] via oblique corrections as they modify the $W, Z$, and photon twopoint functions, and consequently they can be constrained by precision electroweak data. We denote the new contribution to the two-point functions as $\Pi_{V V(0, c)}$ and here we take the opportunity to update the constraints on $\beta_{0}$ and $\beta_{c}$ derived in Ref. [9].

It is easy to notice from the structure of the Lagrangians that the contributions to the $W$ and $Z$ self-energies are con- stant, i.e., they do not depend on the external momentum. Moreover, due to the $S U(2)$ custodial symmetry they are related by

$$
\Pi_{W W(0, c)}=c_{w}^{2} \Pi_{Z Z(0, c)} .
$$

As a consequence the couplings $\beta_{0}$ and $\beta_{c}$ do not contribute to $T=\Delta \rho$ [13]. Equivalently their contribution to $\sin \overline{\theta_{W}}$ vanishes. Moreover, the unbroken $U(1)_{\text {em }}$ symmetry constrains the photon self-energy contribution to be of the form

$$
\Pi_{\gamma \gamma(0, c)}\left(q^{2}\right)=q^{2} \Pi_{\gamma \gamma(0, c)}^{\prime},
$$

where for the anomalous interactions Eq. (9) $\Pi_{\gamma \gamma(0, c)}^{\prime}$ is a constant. This also implies that these anomalous interactions do not modify the running of the electromagnetic coupling. However, both interactions give rise to corrections to $\Delta r$ or, equivalently, to the $S$ and $U$ parameters [13].

Following the standard procedure, we evaluated the vector boson two-point functions using dimensional regularization and subsequently kept only the leading nonanalytic contributions from the loop diagrams to constrain the new interactions - that is, we maintained only the logarithmic terms, dropping all others. The contributions that are relevant for our analysis are easily obtained by the substitution

$$
\frac{2}{4-d} \rightarrow \log \frac{\Lambda^{2}}{\mu^{2}},
$$

where $\Lambda$ is the energy scale which characterizes the appearance of new physics, and $\mu$ is the scale in the process, which we take to be $M_{W}$. After this procedure we obtain

$$
\begin{aligned}
\alpha S= & -4 s_{W}^{2} c_{W}^{2} \Pi_{\gamma \gamma}^{\prime}=-4 s_{W}^{2} c_{W}^{2}\left(\frac { \alpha \beta _ { 0 } M _ { W } ^ { 2 } } { 4 \pi } \left[-\left(1+\frac{1}{2 c_{W}^{4}}\right)\right.\right. \\
& \left.+\frac{3}{2} \ln \left(\frac{\Lambda^{2}}{M_{W}^{2}}\right)+\frac{3}{4 c_{W}^{4}} \ln \left(\frac{\Lambda^{2} c_{W}^{2}}{M_{W}^{2}}\right)\right] \\
& +\frac{\alpha \beta_{c} M_{W}^{2}}{64 \pi}\left[-\left(1+\frac{1}{2 c_{W}^{4}}\right)+6 \ln \left(\frac{\Lambda^{2}}{M_{W}^{2}}\right)\right. \\
& \left.\left.+\frac{3}{c_{W}^{4}} \ln \left(\frac{\Lambda^{2} c_{W}^{2}}{M_{W}^{2}}\right)\right]\right\} \\
\alpha U= & \frac{s_{w}^{2}}{c_{W}^{2}} S .
\end{aligned}
$$

The allowed ranges of $S$ and $U$ depend on the SM parameters. As an illustration of the size of the bounds, we take that for the Higgs boson mass of $M_{H}=300 \mathrm{GeV}$, the $95 \%$ C.L. limits on $S$ and $U$ are $0.34 \leqslant S \leqslant 0.02$ and $-0.13 \leqslant U$ $\leqslant 0.37$ [14]. These bounds can then be translated into the 95\% C.L. limits on $\beta_{0}$ and $\beta_{c}$ presented in Table I.

The LEP Collaborations have directly probed anomalous quartic couplings involving photons. L3 and OPAL have searched for their effects in the reactions $e^{+} e^{-} \rightarrow W^{+} W^{-} \gamma$, 
TABLE I. 95\% C.L. limits on $\beta_{o}$ and $\beta_{c}$ steaming from oblique parameters $S$ and $U$.

\begin{tabular}{lccc}
\hline \hline$\Lambda(\mathrm{TeV})$ & Parameter & $\beta_{0}\left(\mathrm{GeV}^{-2}\right)$ & $\beta_{c}\left(\mathrm{GeV}^{-2}\right)$ \\
\hline 0.5 & $S$ & $(-0.09,1.5) \times 10^{-4}$ & $(-0.29,4.9) \times 10^{-4}$ \\
& $U$ & $(-5.4,1.9) \times 10^{-4}$ & $(-18 ., 6.2) \times 10^{-4}$ \\
2.5 & $S$ & $(-0.04,0.69) \times 10^{-4}$ & $(-0.15,2.5) \times 10^{-4}$ \\
& $U$ & $(-2.5,0.88) \times 10^{-4}$ & $(-9.1,3.2) \times 10^{-4}$ \\
\hline \hline
\end{tabular}

$Z \gamma \gamma$, and $\nu \bar{\nu} \gamma \gamma$, while the ALEPH collaboration has reported results only on the last reaction [4,5]. The combined results for all these searches lead to the following 95\% C.L. direct limits on the quartic vertices [5]

$$
\begin{aligned}
& -4.9 \times 10^{-3} \mathrm{GeV}^{-2}<\beta_{0}<5.6 \times 10^{-3} \mathrm{GeV}^{-2}, \\
& -5.4 \times 10^{-3} \mathrm{GeV}^{-2}<\beta_{c}<9.8 \times 10^{-3} \mathrm{GeV}^{-2} .
\end{aligned}
$$

Another way to constrain the couplings in Eq. (9) is to notice that this effective Lagrangian leads to tree-level unitarity violation in $2 \rightarrow 2$ processes at high energies. In order to extract the unitarity bounds on the anomalous interactions we evaluated the partial wave helicity amplitudes $\left(\tilde{a}_{\nu \mu}^{j}\right)$ for the inelastic scattering $\gamma\left(\lambda_{1}\right) \gamma\left(\lambda_{2}\right) \rightarrow V\left(\lambda_{3}\right) V\left(\lambda_{4}\right)$, with $V$ $=Z$ and $W^{ \pm}$; see Table II. Unitarity requires that [15]

$$
\beta_{V} \sum_{\nu}\left|\tilde{a}_{\nu \mu}^{j}\right|^{2} \leqslant \frac{1}{4}
$$

where $\beta_{V}$ is the velocity of the final state boson in the centerof-mass frame. For the anomalous interactions Eq. (9), the most restrictive bounds come from the $J=0$ partial wave, which read

$$
\begin{aligned}
& \left(\frac{\alpha \beta s}{16}\right)^{2}\left(1-\frac{4 M_{W}^{2}}{s}\right)^{1 / 2}\left(3-\frac{s}{M_{W}^{2}}+\frac{s^{2}}{4 M_{W}^{4}}\right) \leqslant N \quad \text { for } V=W, \\
& \left(\frac{\alpha \beta s}{16 c_{W}^{2}}\right)^{2}\left(1-\frac{4 M_{Z}^{2}}{s}\right)^{1 / 2}\left(3-\frac{s}{M_{Z}^{2}}+\frac{s^{2}}{4 M_{Z}^{4}}\right) \leqslant N \quad \text { for } V=Z,
\end{aligned}
$$

where $\beta=\beta_{0}$ or $\beta_{c}$ and $N=1 / 4$ (4) for $\beta_{0}\left(\beta_{c}\right)$. For instance, unitarity is violated for $\gamma \gamma$ invariant masses above $240 \mathrm{GeV}$ for $\beta_{0}=5.6 \times 10^{-3} \mathrm{GeV}^{-2}$ (one of the present LEP bounds).

These unitarity constraints are of relevance when extracting the bounds on the anomalous couplings at hadron colliders since it is possible to obtain large parton-parton centerof-mass energies, and consequently have a large unitarity violation. The standard procedure to avoid this unphysical behavior of the subprocess cross section and to obtain meaningful limits is to multiply the anomalous couplings by a form factor

$$
\beta_{0, c} \rightarrow\left(1+\frac{M_{\gamma \gamma}^{2}}{\Lambda^{2}}\right)^{-n} \times \beta_{0, c},
$$

TABLE II. $\tilde{a}_{\nu \mu}^{0}$ for the reactions $\gamma\left(\lambda_{1}\right) \gamma\left(\lambda_{2}\right) \rightarrow V\left(\lambda_{3}\right) V\left(\lambda_{4}\right)$, with $V=Z$ and $W^{ \pm}$, where $\mu=\lambda_{1}-\lambda_{2}$ and $\nu=\lambda_{3}-\lambda_{4} . \beta$ stands for $\beta_{0}$ or $\beta_{c}$, and $n_{W^{ \pm}}=1$ (4) for $\beta_{0}\left(\beta_{c}\right)$, and $n_{Z}=c_{W}^{2}\left(4 c_{W}^{2}\right)$ for $\beta_{0}\left(\beta_{c}\right)$.
$\left(\lambda_{1}, \lambda_{2}, \lambda_{3}, \lambda_{4}\right)$ $\tilde{a}_{\nu \mu}^{0}$
$(++++)$ or $(--++)$
$\left(\frac{\alpha s}{16 n_{V}}\right) \beta$
$(++--)$ or $(----)$
$\left(\frac{\alpha s}{16 n_{V}}\right) \beta$
$(++00)$ or $(--00)$
$\left(1-\frac{s}{2 M_{V}^{2}}\right)\left(\frac{\alpha s}{16 n_{V}}\right) \beta$

where $M_{\gamma \gamma}$ is the invariant mass of the photon pair. Of course using this procedure the limits become dependent on the exponent $n$ and the scale $\Lambda$, which is not longer factorizable. In our calculations, we conservatively choose $n=5$ and $\Lambda=0.5 \mathrm{TeV}$ for the Tevatron and $\Lambda=0.5(2.5) \mathrm{TeV}$ for the LHC. In the case of $e^{+} e^{-}$colliders the center-of-mass energy is fixed and the introduction of the form factor Eq. (19) is basically equivalent to a rescaling of the anomalous couplings $\beta_{0, c}$, therefore we should perform this rescaling when comparing results obtained at hadron and $e^{+} e^{-}$colliders. For example, for our choice of $n$ and $\Lambda$ the LEP limits should be weakened by a factor $\simeq 1.6$.

The dynamical effect of the above form factor can be seen in Fig. 1 where we present the normalized invariant mass distribution of the $\gamma \gamma$ pair for the process Eq. (1) at the Tevatron run II and LHC, assuming that only $\beta_{0}$ contributes. As expected, the form factor reduces the number of photon pairs with high invariant mass. Similar behavior is obtained for reaction (2) and for the anomalous $\beta_{c}$ contribution.

\section{SIGNALS AT HADRON COLLIDERS}

In this work we studied reactions (1) and (2) for the Tevatron and LHC, that is, the associated production of a photon pair and a $W^{*}$ or $Z^{*}$ which decay leptonically, as well as the process Eq. (3) only for the LHC since the Tevatron centerof-mass energy is too low for this process to be of any significance. Process Eq. (1) can be used to study the $\gamma \gamma W^{+} W^{-}$vertex while the process Eq. (2) probes the $\gamma \gamma Z Z$ interaction and reaction (3) receives contributions from $\gamma \gamma W^{+} W^{-}$and $\gamma \gamma Z Z$. We evaluated numerically the helicity amplitudes of all the SM subprocesses leading to the $\gamma \gamma l^{ \pm} \nu, \gamma \gamma l^{+} l^{-}$, and $\gamma \gamma j j$ final states where $j$ can be either a gluon, a quark, or an antiquark. The SM amplitudes were generated using Madgraph [16] in the framework of Helas [17] routines. The anomalous interactions arising from the Lagrangian Eq. (9) were implemented as subroutines and were included accordingly. We consistently took into account the effect of all interferences between the anomalous and the SM amplitudes, and did not use the narrow-width approximation for the vector boson propagators.

In the case of the Tevatron collider, we considered the parameters of run I, i.e., $\sqrt{s}=1.8 \mathrm{TeV}$ and an integrated 


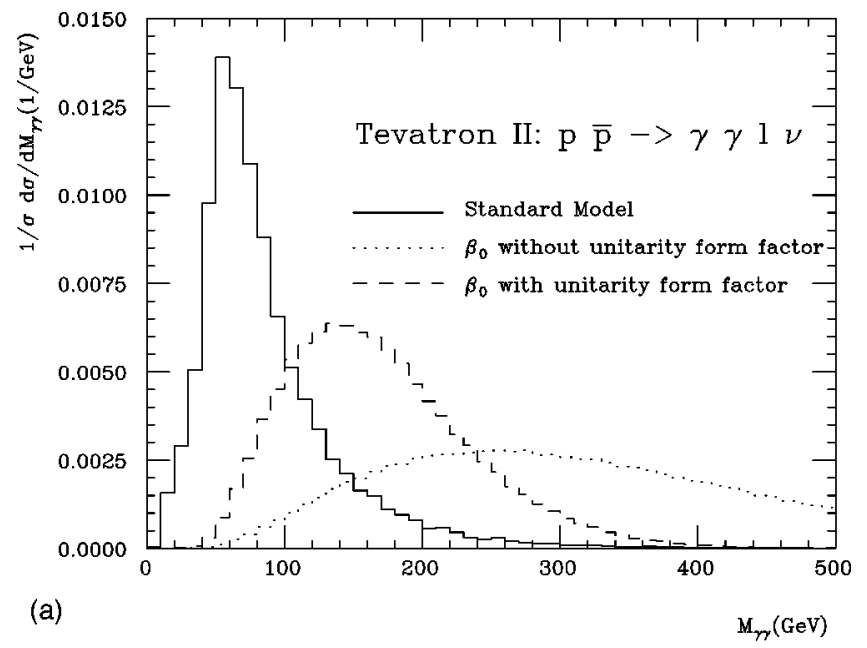

luminosity of $100 \mathrm{pb}^{-1}$. We also investigated the reach of the Tevatron run II assuming $\sqrt{s}=2 \mathrm{TeV}$ and an integrated luminosity of $2 \times 10^{3} \mathrm{pb}^{-1}$. For the LHC, we took a centerof-mass energy of $14 \mathrm{TeV}$ and a luminosity of $10^{5} \mathrm{pb}^{-1}$. In our calculations we used the Martin-Roberts-Sterling set $G$ [MRS $(\mathrm{G})][18]$ of proton structure functions with the factorization scale $Q^{2}=\hat{s}$.

We started our analysis of the processes, Eqs. (1) and (2), imposing a minimal set of cuts to guarantee that the photons and charged leptons are detected and isolated from each other:

$$
\begin{aligned}
p_{T}^{(l, \nu)} & \geqslant 20 \quad(25) \mathrm{GeV} \text { for } l=e(\mu) \\
E_{T}^{\gamma} & \geqslant 20 \mathrm{GeV} \\
\left|\eta_{\gamma, e}\right| & \leqslant 2.5 \\
\left|\eta_{\mu}\right| & \leqslant 1.0 \\
\Delta R_{i j} & \geqslant 0.4
\end{aligned}
$$

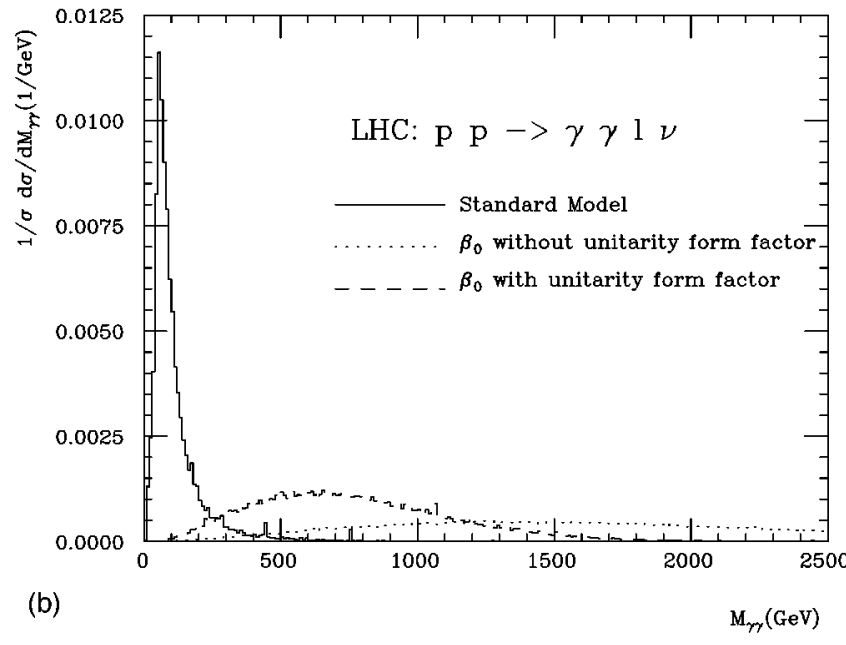

FIG. 1. Normalized invariant mass distribution of the $\gamma \gamma$ pair for the reaction $p+p(\bar{p}) \rightarrow \gamma+\gamma+\left(W^{*} \rightarrow\right) l+\nu$ at Tevatron run II (a) and LHC (b). The solid histogram represents the SM contribution while dashed (dotted) histograms are the anomalous $\beta_{0}$ contribution with (without) unitarity form factor. We chose $n=5$ and $\Lambda$ $=0.5(2.5) \mathrm{TeV}$ for the Tevatron (LHC). where $i$ and $j$ stand for the final photons and charged leptons. For the $\gamma \gamma l \nu$ final state, we also imposed a cut of the transverse mass of the $l \nu$ pair $\left(M_{T}^{l \nu}\right)$ :

$$
65 \mathrm{GeV} \leqslant M_{T}^{l \nu} \leqslant 100 \mathrm{GeV} .
$$

In the case of $\gamma \gamma l^{+} l^{-}$production, we required the tag of a $Z$ decaying leptonically imposing that

$$
75 \mathrm{GeV} \leqslant M^{l l} \leqslant 105 \mathrm{GeV},
$$

where $M^{l l}$ is the invariant mass of the lepton pair. In our calculations, we have also taken into account the detection efficiency of the final state particles. We assumed an $85 \%$ detection efficiency of isolated photons, electrons, and muons. Therefore, the efficiency for reconstructing the final state $\gamma \gamma l \nu$ is $61 \%$ while the efficiency for $\gamma \gamma l^{+} l^{-}$is $52 \%$.

Considering the cuts Eqs. (20), (21), and (22), and the detection efficiencies discussed above, the SM prediction for

TABLE III. SM cross sections after the cuts. We applied the cuts Eqs. (20)-(22) to the $l \nu \gamma \gamma$ and $l^{+} l^{-} \gamma \gamma$ processes while we used the cuts Eqs. (20) and Eqs. (26), (27) to the $\gamma \gamma j j$ final state. We present between parenthesis the Tevatron II results after we included the additional cut Eq. (23) for $l \nu \gamma \gamma$ and $l^{+} l^{-} \gamma \gamma$ productions. In the case of $j j \gamma \gamma$ production at LHC, we exhibit between parenthesis (brackets) the results after cuts Eq. (28) for $\Lambda=0.5$ (2.5) TeV.

\begin{tabular}{lccc}
\hline \hline Collider & Process & Cross section $(\mathrm{pb})$ & Number of events \\
\hline Tevatron I & $p \bar{p} \rightarrow l^{ \pm} \nu_{l^{ \pm}} \gamma \gamma$ & $1.93 \times 10^{-4}$ & $1.93 \times 10^{-2}$ \\
& $p \bar{p} \rightarrow l^{+} l^{-} \gamma \gamma$ & $1.58 \times 10^{-4}$ & $1.58 \times 10^{-2}$ \\
Tevatron II & $p \bar{p} \rightarrow l^{ \pm} \nu_{l^{ \pm}} \gamma \gamma$ & $2.13 \times 10^{-4}\left(7.89 \times 10^{-6}\right)$ & $0.43\left(1.58 \times 10^{-2}\right)$ \\
& $p \bar{p} \rightarrow l^{+} l^{-} \gamma \gamma$ & $1.77 \times 10^{-4}\left(5.90 \times 10^{-6}\right)$ & $0.35\left(1.18 \times 10^{-2}\right)$ \\
LHC & $p p \rightarrow l^{ \pm} \nu_{l^{ \pm}} \gamma \gamma$ & $1.08 \times 10^{-3}\left(1.32 \times 10^{-5}\right)$ & $108(1.3)$ \\
& $p p \rightarrow l^{+} l^{-} \gamma \gamma$ & $6.45 \times 10^{-4}\left(4.25 \times 10^{-6}\right)$ & $65(0.43)$ \\
& $p p \rightarrow j j \gamma \gamma$ & $3.19 \times 10^{-2}\left(6.28 \times 10^{-3}\right)\left[1.12 \times 10^{3}\right]$ & $3190(628)[112]$ \\
\hline \hline
\end{tabular}




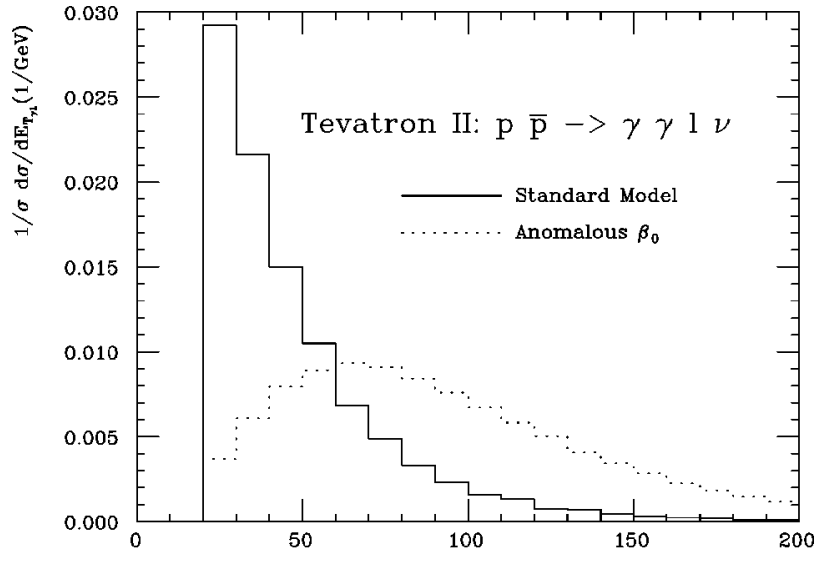

(a)

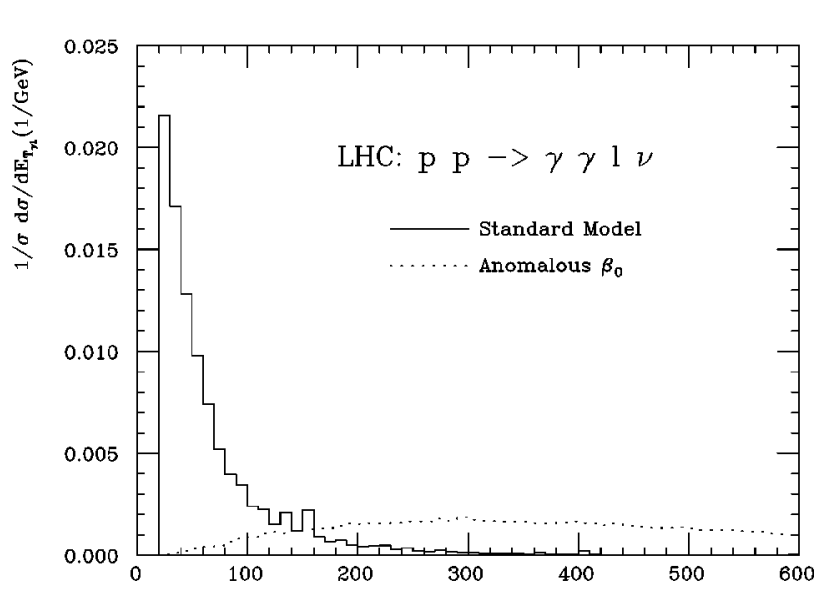

(b)

$\mathrm{E}_{\mathrm{T}_{\mathbf{n}}}(\mathrm{GeV})$

FIG. 2. Normalized transverse energy distribution of the most energetic photon for the reaction Eq. (1) at Tevatron run II (a) and LHC (b). The solid histogram represents the SM contribution while the dotted one is the anomalous $\beta_{0}$ contribution.

the cross sections and expected number of events of the processes Eqs. (1) and (2) are presented in Table III. As we can see, the above basic cuts are enough to eliminate the SM background at the Tevatron run I, however, further cuts are needed to control the background at the Tevatron run II and LHC.

In order to reduce the SM background for the Tevatron run II and LHC, we analyzed a few kinematical distributions. The most significant difference between the SM and anomalous predictions appears in the transverse energy of the photons, which is shown in Fig. 2 for the reaction Eq. (1) and $\beta_{0} \neq 0$. Similar behavior is obtained for the reaction (2) and for the anomalous $\beta_{c}$ contribution. Therefore, we tightened the cut on the transverse energy of the final photons, as suggested by Fig. 2, to enhance the significance of the anomalous contribution.

$$
E_{T}^{\gamma_{1(2)}} \geqslant 75 \text { (50) GeV for Tevatron run II }
$$

and

$$
E_{T}^{\gamma_{1(2)}} \geqslant 200 \text { (100) GeV for LHC. }
$$

The effect of these cuts can be seen in Table III where we display the new cross sections and expected number of events in parenthesis. As we can see, no SM event is expected at the Tevatron run II after this new cut, while very few events survive at the LHC.

We parametrized the cross sections for processes (1) and (2) after cuts Eqs. (20)-(23) as

$$
\sigma \equiv \sigma_{\mathrm{sm}}+\beta \sigma_{\text {inter }}+\beta^{2} \sigma_{\text {ano }}
$$

where $\sigma_{\mathrm{sm}}, \sigma_{\text {inter }}$, and $\sigma_{\text {ano }}$ are, respectively, the SM cross section, interference between the SM and the anomalous contribution, and the pure anomalous cross section. $\beta$ stands for $\beta_{0}$ or $\beta_{c}$. The results for $\sigma_{\mathrm{sm}}, \sigma_{\text {inter }}$, and $\sigma_{\text {ano }}$ are presented in Table IV.

Process (3) receives contributions from $W^{*}$ and $Z^{*}$ productions and their subsequent decay into jets, as well as from vector boson fusion $(\mathrm{VBF})$

$$
p+p \rightarrow q+q+\left(W^{*}+W^{*} \text { or } Z^{*}+Z^{*}\right) \rightarrow q+q+\gamma+\gamma
$$

TABLE IV. Results for $\sigma_{\text {sm }}, \sigma_{\text {inter }}$, and $\sigma_{\text {ano }}$; see Eq. (24). $\sigma_{\text {inter }}$ and $\sigma_{\text {ano }}$ are obtained for the anomalous coupling $\beta_{0}\left(\beta_{c}\right)$ in units of $\mathrm{GeV}^{-2}$. We considered $n=5$ and different values of $\Lambda$; see Eq. (19).

\begin{tabular}{lcccc}
\hline \hline Collider & Process & $\sigma_{\mathrm{sm}}(\mathrm{pb})$ & $\sigma_{\text {inter }}\left(\mathrm{pb} \times \mathrm{GeV}^{2}\right)$ for $\beta_{0}\left(\beta_{c}\right)$ & $\sigma_{\text {ano }}\left(\mathrm{pb} \times \mathrm{GeV}^{4}\right)$ for $\beta_{0}\left(\beta_{c}\right)$ \\
\hline Tevatron I & $p \bar{p} \rightarrow l^{ \pm} \nu_{l^{ \pm}} \gamma \gamma$ & $1.93 \times 10^{-4}$ & $5.09(2.58) \times 10^{-3}$ & $15.0(5.50)$ \\
$\Lambda=0.5 \mathrm{TeV}$ & $p \bar{p} \rightarrow l^{+} l^{-} \gamma \gamma$ & $1.58 \times 10^{-4}$ & $7.18(1.22) \times 10^{-3}$ & $3.63(1.37)$ \\
Tevatron II & $p \bar{p} \rightarrow l^{ \pm} \nu_{l^{ \pm}} \gamma$ & $7.89 \times 10^{-6}$ & $1.20(1.03) \times 10^{-3}$ & $6.21(2.92)$ \\
$\Lambda=0.5 \mathrm{TeV}$ & $p \bar{p} \rightarrow l^{+} l^{-} \gamma \gamma$ & $5.90 \times 10^{-6}$ & $1.38(0.36) \times 10^{-3}$ & $1.78(0.86)$ \\
LHC & $p p \rightarrow l^{ \pm} \nu_{l^{ \pm}} \gamma \gamma$ & $1.32 \times 10^{-5}$ & $3.13(3.97) \times 10^{-4}$ & $6.79(59.2)$ \\
$\Lambda=0.5 \mathrm{TeV}$ & $p p \rightarrow l^{+} l^{-} \gamma \gamma$ & $4.25 \times 10^{-6}$ & $6.06(0.49) \times 10^{-4}$ & $4.82(18.5)$ \\
& $p p \rightarrow j j \gamma \gamma$ & $6.28 \times 10^{-3}$ & - & $1.02 \times 10^{4}\left(7.56 \times 10^{2}\right)$ \\
$\mathrm{LHC}$ & $p p \rightarrow l^{ \pm} \nu_{l^{ \pm}} \gamma \gamma$ & $1.32 \times 10^{-5}$ & $1.17(22.4) \times 10^{-3}$ & $5570(2900)$ \\
$\Lambda=2.5 \mathrm{TeV}$ & $p p \rightarrow l^{+} l^{-} \gamma \gamma$ & $4.25 \times 10^{-6}$ & $1.15(1.08) \times 10^{-2}$ & $3980(1390)$ \\
& $p p \rightarrow j j \gamma \gamma$ & $1.12 \times 10^{-3}$ & - & $1.07 \times 10^{7}\left(7.34 \times 10^{5}\right)$ \\
\hline \hline
\end{tabular}


The signal for hadronic decays of W's and $Z$ 's is immersed in a huge QCD background. Therefore, we tuned our cuts in order to extract the VBF production of photon pairs since it presents two very energetic forward jets that can be used to efficiently tag the events. In our analyses, we required that the photons satisfy

$$
\begin{aligned}
E_{T}^{\gamma_{1(2)}}>50(25) \mathrm{GeV}, \\
\left|\eta_{\gamma_{(1,2)}}\right|<5.0
\end{aligned}
$$

while the jets should comply with

$$
\begin{gathered}
p_{T}^{j_{1(2)}}>40 \quad(20) \mathrm{GeV}, \\
\left|\eta_{j_{(1,2)}}\right|<5.0, \\
\left|\eta_{j_{1}}-\eta_{j_{2}}\right|>4.4, \\
\eta_{j_{1}} \cdot \eta_{j_{2}}<0, \\
\min \left\{\eta_{j_{1}}, \eta_{j_{1}}\right\}+0.7<\eta_{\gamma_{(1,2)}}<\max \left\{\eta_{j_{1}}, \eta_{j_{1}}\right\}-0.7, \\
\Delta R_{j j}>0.7, \\
\Delta R_{j \gamma}>0.7 .
\end{gathered}
$$

Assuming an $85 \%$ detection efficiency of isolated photons, the efficiency for reconstructing the final state jet + jet $+\gamma$ $+\gamma$ is $72 \%$. Table III also contains the SM cross section for the VBF production of photon pairs taking into account the above cuts. As we can see, the VBF reaction possesses much higher statistics than the production of photon pairs associated to leptons. In order to enhance the VBF signal for the anomalous couplings we studied a few kinematical distributions and found that the most significant difference between the signal and SM background occurs in the diphoton invariant mass spectrum; see Fig. 3. Thusly, we imposed the following additional cuts:

$$
\begin{gathered}
200 \text { (400) } \mathrm{GeV} \leqslant M_{\gamma \gamma} \leqslant 700 \text { (2500) } \mathrm{GeV} \text { for } \\
\Lambda=500(2500) \mathrm{GeV} \text {. }
\end{gathered}
$$

This cut reduces the SM background cross section by a factor of at least 5; see Table III where we also present the signal cross section after cuts for $\Lambda=500$ and $2500 \mathrm{GeV}$. The results for $\sigma_{\mathrm{sm}}$ and $\sigma_{\mathrm{ano}}$ of Eq. (24) are presented in Table IV. Since the interference between the SM and the anomalous contribution is negligible in this case, we do not present the results for $\sigma_{\text {inter }}$.

Taking into account the integrated luminosities of the Tevatron and LHC and the results shown in Table IV, we evaluated the potential 95\% C.L. limits on $\beta_{0}$ and $\beta_{c}$ in the case where there is no deviation from the SM predictions; see Table V. We also exhibit in this table our choice for the scale $\Lambda$ appearing in the form factor. Therefore, at the Tevatron, the most restrictive constraints are obtained from the reaction Eq. (1) for $\beta_{0}$ and $\beta_{c}$. Combining both reactions we
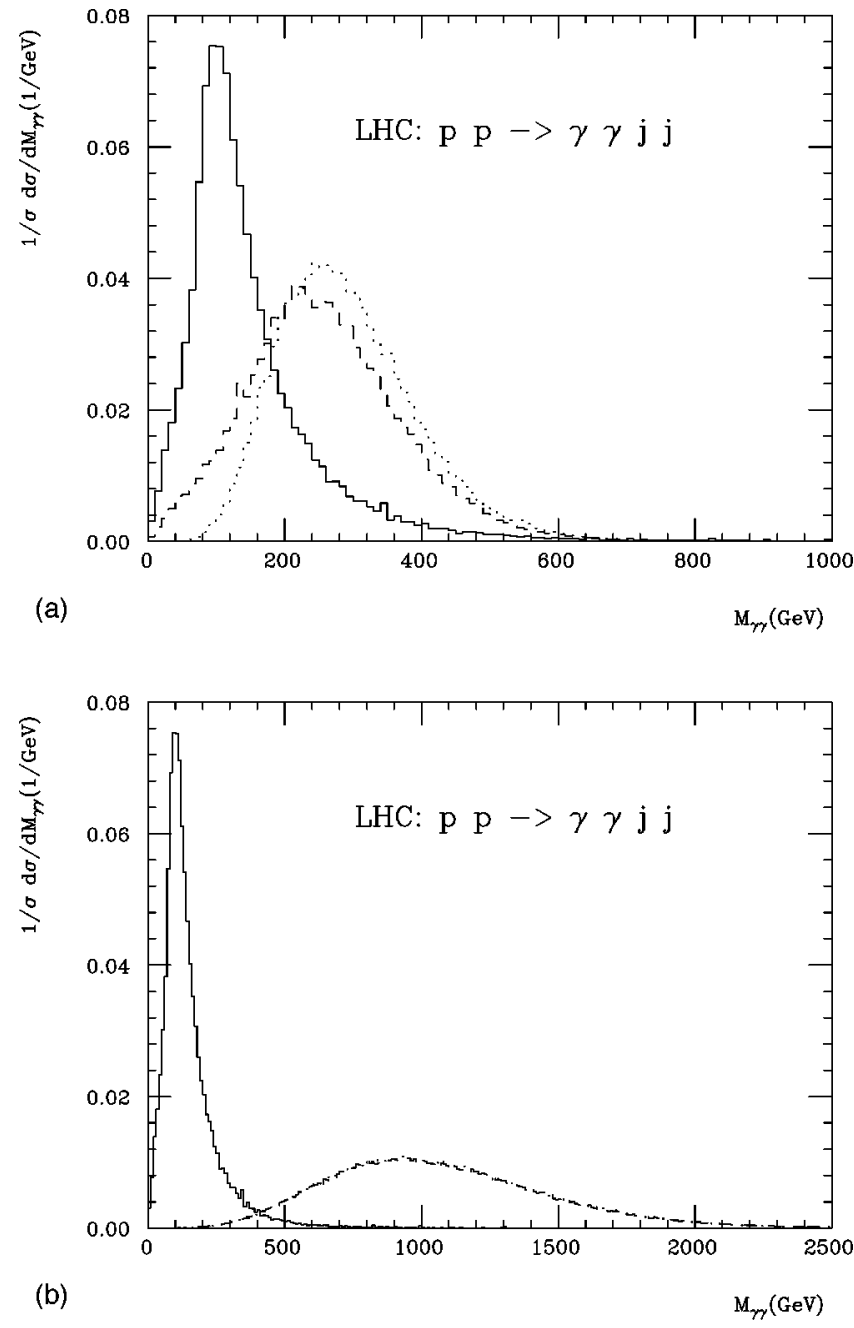

FIG. 3. Normalized invariant mass distribution of the $\gamma \gamma$ pair for the reaction $p+p \rightarrow \gamma+\gamma+$ jet + jet at LHC. The solid histogram represents the SM contribution while dotted (dashed) histograms are the anomalous $\beta_{0}\left(\beta_{c}\right)$ contribution with unitarity form factor. We chose $n=5$ and (a) $\Lambda=0.5 \mathrm{TeV}$ and (b) $\Lambda=2.5 \mathrm{TeV}$.

are able to impose a 95\% C.L. limit $\left|\beta_{0, c}\right| \lesssim 1.5$ $\times 10^{-2} \mathrm{GeV}^{-2}$ at the Tevatron run II, which is of the same order as the direct bounds coming from LEPII. On the other hand, the most stringent limits at the LHC will come from the photon pair production via VBF, whose bounds are a factor of 5-10 stronger than the ones coming from the reactions Eqs. (1) and (2). This general statement does not seem to apply for the limits on $\beta_{c}$ with $\Lambda=500 \mathrm{GeV}$, which is more strongly constrained by the process Eq. (1). This is not surprising because, for the reactions Eqs. (1) and (2), the set of cuts Eq. (23) leave the $\beta_{c}$ signal practically unaffected, i.e., this set of general cuts is particularly optimum for this coupling and reactions. This is also the reason why the derived limits on $\beta_{c}$ are better than the limits for $\beta_{0}$ only for this case.

\section{SUMMARY AND CONCLUSIONS}

We are just beginning to test the SM predictions for the quartic vector boson interactions. Because of the limited 
TABLE V. 95\% C.L. limits on $\beta_{0}$ and $\beta_{c}$ that can be obtained at the Tevatron and LHC assuming that no deviation from the SM predictions is observed. We considered $n=5$ and different values of $\Lambda$; see Eq. (19).

\begin{tabular}{lccc}
\hline \hline Collider & Process & $\beta_{0}\left(\mathrm{GeV}^{-2}\right)$ & $\beta_{c}\left(\mathrm{GeV}^{-2}\right)$ \\
\hline Tevatron I & $p \bar{p} \rightarrow l^{ \pm} \nu_{l^{ \pm}} \gamma \gamma$ & $(-4.5,4.4) \times 10^{-2}$ & $(-7.4,7.4) \times 10^{-2}$ \\
& $p \bar{p} \rightarrow l^{+} l^{-} \gamma \gamma$ & $(-9.2,9.0) \times 10^{-2}$ & $(-15 ., 15.) \times 10^{-2}$ \\
$\Lambda=0.5 \mathrm{TeV}$ & Combined & $(-4.0,4.0) \times 10^{-2}$ & $(-6.6,6.5) \times 10^{-2}$ \\
Tevatron II & $p \bar{p} \rightarrow l^{ \pm} \nu_{l^{ \pm}} \gamma \gamma$ & $(-1.6,1.5) \times 10^{-2}$ & $(-2.3,2.2) \times 10^{-2}$ \\
& $p \bar{p} \rightarrow l^{+} l^{-} \gamma \gamma$ & $(-2.9,2.9) \times 10^{-2}$ & $(-4.2,4.1) \times 10^{-2}$ \\
$\Lambda=0.5 \mathrm{TeV}$ & Combined & $(-1.4,1.3) \times 10^{-2}$ & $(-2.0,2.0) \times 10^{-2}$ \\
LHC & $p p \rightarrow l^{ \pm} \nu_{l^{ \pm}} \gamma \gamma$ & $(-2.2,2.1) \times 10^{-3}$ & $(-7.4,7.3) \times 10^{-4}$ \\
& $p p \rightarrow l^{+} l^{-} \gamma \gamma$ & $(-2.4,2.3) \times 10^{-3}$ & $(-12 ., 12.) \times 10^{-4}$ \\
$\Lambda=0.5 \mathrm{TeV}$ & $p p \rightarrow j j \gamma \gamma$ & $(-2.2,2.2) \times 10^{-4}$ & $(-8.0,8.0) \times 10^{-4}$ \\
LHC & $p p \rightarrow l^{ \pm} \nu_{l^{ \pm}} \gamma \gamma$ & $(-7.6,7.6) \times 10^{-5}$ & $(-11 ., 10.) \times 10^{-5}$ \\
& $p p \rightarrow l^{+} l^{-} \gamma \gamma$ & $(-8.2,7.9) \times 10^{-5}$ & $(-14 ., 13.) \times 10^{-5}$ \\
$\Lambda=2.5 \mathrm{TeV}$ & $p p \rightarrow j j \gamma \gamma$ & $(-4.4,4.4) \times 10^{-6}$ & $(-1.7,1.7) \times 10^{-5}$ \\
\hline \hline
\end{tabular}

available center-of-mass energy, the first couplings to be studied contain two photons, and just at the LHC and the Next Linear Collider (NLC) we will be able to probe $V V V V$ ( $V=W$ or $Z$ ) vertices [19]. In this work we analyzed the production of photon pairs in association with $l^{ \pm} \nu, l^{+} l^{-}$, or $j j$ in hadron colliders. These processes violate unitarity at high energy; therefore, we cut off the growth of the subprocess cross section via the introduction of form factors which enforce unitarity and render the calculation meaningful.

We showed that the study of the processes Eqs. (1) and (2) at Tevatron run I lead to constraints on the quartic anomalous couplings that are a factor of 4 weaker than the presently available bounds derived from LEPII data. On the other hand, the Tevatron run II has the potential to probe the quartic anomalous interactions at the same level of LEPII. An important improvement on the bounds on the genuine quartic couplings will be obtained at the LHC collider where, for $\Lambda=2.5 \mathrm{TeV}$, a limit of $\left|\beta_{0, c}\right| \lesssim 10^{-5} \mathrm{GeV}^{-2}$ will be reached. Therefore, the direct limits on the anomalous inter- action steaming from LHC will be stronger than the ones coming from the precise measurements at the $Z$ pole. It is interesting to note that the LHC will lead to limits that are similar to the ones attainable at an $e^{+} e^{-}$collider operating at $\sqrt{s}=500 \mathrm{GeV}$ with a luminosity of $300 \mathrm{pb}^{-1}$, which are $\left|\beta_{0, c}\right| \lesssim 3 \times 10^{-5} \mathrm{GeV}^{-2}[10]$.

In conclusion, the LHC will be able to impose quite important limits on genuine quartic couplings studying the $\gamma \gamma l^{+} l^{-}, \gamma \gamma l \nu$, and $\gamma \gamma j j$ productions.

\section{ACKNOWLEDGMENTS}

This work was supported by Conselho Nacional de Desenvolvimento Científico e Tecnológico ( $\mathrm{CNPq})$, by Fundação de Amparo à Pesquisa do Estado de São Paulo (FAPESP), by Programa de Apoio a Núcleos de Excelência (PRONEX), by the Spanish DGICYT under Grant Nos. PB98-0693 and PB97-1261, by the Generalitat Valenciana under Grant No. GV99-3-1-01, and by the TMR network Grant No. ERBFMRXCT960090 of the European Union.
[1] For a review see: H. Aihara et al., Anomalous Gauge Boson Interactions, in Electroweak Symmetry Breaking and New Physics at the TeV Scale, edited by T. Barklow, S. Dawson, H. Haber and J. Seigrist (World Scientific, Singapore, 1996), p. 488, hep-ph/9503425.

[2] CDF Collaboration, K. Gounder, hep-ex/9903038; DØ Collaboration, B. Abbott et al., Phys. Rev. D 62, 052005 (2000).

[3] ALEPH Collaboration, R. Barate et al., Phys. Lett. B 462, 389 (1999); DELPHI Collaboration, P. Abreu et al., ibid. 459, 382 (1999); L3 Collaboration, M. Acciarri et al., ibid. 467, 171 (1999); OPAL Collaboration, G. Abbiendi et al., Eur. Phys. J. C 8, 191 (1999)

[4] OPAL Collaboration, G. Abbiendi et al., Phys. Lett. B 471, 293 (1999); L3 Collaboration, M. Acciarri et al., ibid. 478, 39 (2000); 490, 187 (2000).
[5] S. Spagnolo, presented at the XXXth International Conference on High Energy Physics, Osaka 2000, http:// ichep2000.hep.sci.osaka-u.ac.jp/scan/0727/pa05/spagnolo/

[6] A. Hill and J.J. van der Bij, Phys. Rev. D 36, 3463 (1987); R. Casalbuoni et al., Nucl. Phys. B282, 235 (1987); Phys. Lett. 155B, 95 (1985); S. Godfrey, in Quartic Gauge Boson Couplings, in AIP Conf. Proc. No. 350, edited by U. Baur, S. Errede, and T. Muller (AIP, New York, 1996), p. 209, hep-ph/9505252.

[7] G. Bélanger and F. Boudjema, Phys. Lett. B 288, 210 (1992).

[8] O.J.P. Éboli, M.B. Magro, P.G. Mercadante, and S.F. Novaes, Phys. Rev. D 52, 15 (1995).

[9] O.J.P. Éboli, M.C. Gonzalez-Garcia, and S.F. Novaes, Nucl. Phys. B411, 381 (1994).

[10] G. Bélanger and F. Boudjema, Phys. Lett. B 288, 201 (1992); 
W.J. Stirling and A. Werthenbach, ibid. 466, 369 (1999); W.J. Stirling and A. Werthenbach, Eur. Phys. J. C 14, 103 (2000); G. Belanger, F. Boudjema, Y. Kurihara, D. Perret-Gallix, and A. Semenov, ibid. 13, 283 (2000).

[11] P.J. Dervan, A. Signer, W.J. Stirling, and A. Werthenbach, J. Phys. G 26, 607 (2000).

[12] C.P. Burgess and D. London, Phys. Rev. D 48, 4337 (1993).

[13] M.E. Peskin and T. Takeuchi, Phys. Rev. Lett. 65, 964 (1990); Phys. Rev. D 46, 381 (1992).

[14] Particle Data Group, D.E. Groom et al., Eur. Phys. J. C 15, 1 (2000).

[15] U. Baur and D. Zeppenfeld, Nucl. Phys. B308, 127 (1988).

[16] T. Stelzer and W.F. Long, Comput. Phys. Commun. 81, 357 (1994).

[17] H. Murayama, I. Watanabe, and K. Hagiwara, KEK Report
No. 91-11.

[18] A.D. Martin, W.J. Stirling, and R.G. Roberts, Phys. Lett. B 354, 155 (1995).

[19] O.J.P. Éboli, M.C. Gonzalez-Garcia, and J.K. Mizukoshi, Phys. Rev. D 58, 034008 (1998); T. Han, H.-J. He, and C.-P. Yuan, Phys. Lett. B 422, 294 (1998); V. Barger, K. Cheung, T. Han, and R.J.N. Philips, Phys. Rev. D 52, 3815 (1995); E.E. Boos, H.-J. He, W. Kilian, A. Pukhov, C.-P. Yuan, and P.M. Zerwas, ibid. 57, 1553 (1998); J. Bagger, S. Dawson, and G. Valencia, Nucl. Phys. B399, 364 (1993); A. Dobado, D. Espriu, and M.J. Herrero, Z. Phys. C 50, 205 (1991); A.S. Belyaev et al., Phys. Rev. D 59, 015022 (1999); A. Dobado and M.T. Urdiales, Z. Phys. C 17, 965 (1996); J.R. Pelaez, Phys. Rev. D 55, 4193 (1997). 